\title{
Etude des quantités ingérées lors des grands repas chez des moutons recevant des fourrages ensilés
}

\author{
J.P. DULPHY \\ avec la collaboration technique de J.M. BOISSAU, H. BOUSQUET, \\ Jacqueline JAMOT, Marie JAILLER et L. L'HOTELIER \\ I.N.R.A., Laboratoire des Aliments \\ C.R.Z.V. de Theix, F 63122 Ceyrat
}

\section{Résumé}

Pour 109 ensilages (graminées fourragères, trèfle violet, luzerne et maïs), nous avons mesuré, chez le mouton, les quantités de matière sèche ingérées lors des grands repas suivant la distribution de l'aliment. Ces quantités sont très faibles par rapport aux quantités ingérées lors des grands repas sous forme de fourrage vert : 40 à $60 \mathrm{p}$. 100, alors que la vitesse d'ingestion ne semble pas modifiée par rapport à celle des fourrages verts.

Pour des qualités de conservation proches, la finesse de hachage influe peu sur les quantités ingérées. Par contre, les légumineuses donnent des résultats beaucoup plus élevés que pour les graminées fourragères et le maïs. Enfin la diminution de la qualité de conservation entraîne une baisse des quantités ingérées par grands repas.

Ces résultats sont discutés en considérant tout d'abord les ensilages bien conservés pour lesquels la présence d'acide lactique pourrait expliquer le phénomène de rassasiement rapide observé. Ensuite, lorsque la qualité de conservation se dégrade, l'augmentation des teneurs en acides gras volatils, en particulier en acide acétique, pourrait expliquer un renforcement du phénomène avec, au départ, une action des produits de fermentation sur le goût, l'odorat et/ou des chimiorécepteurs ruminaux et duodénaux.

Le phénomène de rassasiement rapide observé chez le mouton peut expliquer partiellement le niveau plus faible des quantités d'ensilage ingérées par rapport à celles de fourrage vert. L'animal compense cependant en ingérant plus d'ensilage que de fourrage vert lors des petits repas, ce qui indique que l'action de certains facteurs intervenant lors des grands repas ait diminué au niveau de la journée.

Mots clés : Ensilage, ovins, ingestion, rassasiement, repas.

\section{Introduction}

Les ruminants consommant des aliments grossiers, riches en parois végétales, sont soumis à une régulation de leur ingestion de type physique (BALCH \& CAMPLING, 1962), mais de nombreux facteurs peuvent intervenir et perturber ce schéma classi- 
que : facteurs oropharingés, facteurs gastrointestinaux (acides acétique et propionique), métabolites, hormones, etc. (Baile \& Della-Ferra, 1981). Dans ces conditions, l'animal mange en général moins que ne le permettrait la capacité «physique » de son tractus digestif. Cela est particulièrement vrai pour les ensilages d'herbe (DEMARQUILLY, 1973 ; DULPHY, 1979).

L'ingestion des aliments est répartie au cours de la journée selon des modalités variables avec le type d'animal et le type d'aliment (DulPhy, Remond \& Theriez, 1979). Lorsque l'aliment est distribué on observe, après chaque distribution, un repas relativement important ("grand repas») et, au cours de la journée, des petits repas (Dulphy, Michalet-Doreau \& Demarquilly, 1984). La régulation journalière de l'ingestion passe donc par le contrôle de l'importance de chacun des repas. Or les travaux que nous avons entrepris sur la régulation des quantités d'ensilage ingérées par les ruminants ont mis en évidence que les quantités de matière sèche ingérées lors des grands repas étaient faibles pour les ovins. Cette faiblesse pourrait expliquer en partie le fait que les moutons ingèrent, par jour, moins de matière sèche sous forme d'ensilage que de fourrage vert, bien qu'une compensation partielle se fasse grâce à une augmentation du nombre de repas au cours de la journée (DuLPHY, Michalet-Doreau \& Demarquilly, 1984). Partant de cette hypothèse nous avons essayé de mieux connaître quels étaient les facteurs, liés aux ensilages, responsables du rassasiement rapide des animaux.

Dans un cadre général, d'après Forbes (1979) quatre signaux importants peuvent intervenir, au cours des repas, pour faire cesser l'ingestion chez les ruminants : la distension des parois du tube digestif, la concentration ruminale en acide acétique, la concentration duodénale en acide lactique et l'augmentation de l'acide propionique au niveau du foie. Les effets de l'acide acétique ont été largement étudiés (BaIle \& MAYER, 1969 ; BAILE \& Forbes, 1974) et on sait que l'ingestion des ensilages est négativement reliée à la teneur en cet acide (WILKINs et al., 1971). Bien que moins étudié, l'acide lactique a aussi un effet sur les quantités ingérées (Morgan \& LeSTRANGe, 1976) ; cet acide est présent en quantité parfois élevée dans les ensilages. Quant aux deux autres signaux, ils ont moins de chance d'être mis en jeu car les bons ensilages ne contiennent pas d'acide propionique, mais entraînent un rassasiement rapide (DulPhy, Michalet-Doreau \& Demarquilly, 1984). En outre, la capacité totale du rumen ne semble pas utilisée pour les ensilages (Dulphy, BECHET \& Thomson, 1975). Nous avons alors supposé que l'acide acétique et l'acide lactique des ensilages, par leur arrivée rapide dans le rumen, lors des grands repas, pourraient expliquer nos observations.

Pour cette étude nous avons donc comparé des ensilages différents par leur nature (graminées, légumineuses, maïs), la longueur de leurs brins, qui est un facteur important de régulation de leurs quantités ingérées, et surtout leur qualité de conservation (teneurs en acide acétique et lactique) et mesuré comment variait l'importance des grands repas. L'influence de la teneur en ammoniac des ensilages a aussi été examinée car ce facteur est connu pour agir négativement sur les quantités d'ensilage ingérées (WILkins et al., 1971). Lorsque cela a été possible, les ensilages ont été comparés aux fourrages verts initiaux. 


\section{Matériel et Méthodes}

A partir de 20 fourrages sur pied (ray-grass anglais, ray-grass d'Italie, fléole, fétuque élevée, trèfle violet, luzerne) ainsi qu'à partir de 14 maïs, 109 ensilages ont été préparés. Ces fourrages ont été récoltés avec trois machines différentes :

- une machine à fléaux (brins longs de 10 à $25 \mathrm{~cm}$ );

- une machine à plateau hacheur (brins moyens de 5 à $15 \mathrm{~cm}$ ) ;

- une machine à tambour hacheur (brins courts de 1 à $2 \mathrm{~cm}$ ).

Une partie des ensilages à brins moyens ou longs a également été étudiée après passage dans un hache-paille à la sortie du silo (brins de 2 à $3 \mathrm{~cm}$; ensilages dits * hachés ») (Dulphy \& Demarquilly, 1973). La répartition de l'ensemble des ensilages est donnée dans le tableau 1. Tous ces fourrages ont été conservés dans des petits silos en plastique ou en butyl de $4 \mathrm{~m}^{3}$. Certains ont reçu un conservateur (acide formique, 3,5 1/t ; kofasil, $7 \mathrm{~kg}$ /tonne).

\section{Tableau 1}

Répartition des ensilages étudiés.

List of studied silages.

\begin{tabular}{c|c|c|c}
\hline \hline $\begin{array}{c}\text { Espèce végétale } \\
\text { Forage varieties }\end{array}$ & $\begin{array}{c}\text { Machine de récolte - Harvester } \\
\text { ì fléaux } \\
\text { flail-harvester }\end{array}$ & $\begin{array}{c}\text { à plateau hacheur } \\
\text { double-chop } \\
\text { harvester }\end{array}$ & $\begin{array}{c}\text { à tambour-hacheur } \\
\text { precision chop } \\
\text { harvester }\end{array}$ \\
\hline Graminées - Grasses ..... & $2+2 \mathrm{H}$ & $5+5 \mathrm{H}$ & 44 \\
Trèfle violet - Red clover .. & $2+2 \mathrm{H}$ & $2+2 \mathrm{H}$ & 2 \\
Luzerne - Lucerne ......... & $3+2 \mathrm{H}$ & 4 & 18 \\
Maïs - Maize ............ & - & - & 14 \\
\hline
\end{tabular}

H : Ensilage haché à la sortie du silo - Silage chopped after unioading from silo.

Les fourrages verts, sauf les maïs et une graminée, ont été distribués à volonté à des béliers castrés de race Texel dans les conditions décrites précédemment par Demarquilly (1973) et Dulphy, Michalet-Doreau \& Demarquilly (1984); leurs quantités ingérées par jour ont été mesurées.

Les ensilages ont été distribués durant l'hiver à des béliers différents, mais comparables. Les mêmes mesures que précédemment ont été faites.

Les quantités ingérées par grands repas (pour tous les ensilages ainsi que pour 13 fourrages verts, qi pour chacun des 2 grands repas) ont été mesurées après avoir pesé ce qui restait dans l'auge une fois le grand repas terminé. Le début de ces grands repas était fixé à 8 et à 16 heures, heure de distribution des fourrages aux moutons. Pour 30 ensilages nous avons déterminé précisément la fin des grands repas en 
suivant les activités alimentaires des animaux par la méthode de Ruckebusch (1963) qui permet d'enregistrer et de décrire le comportement alimentaire et mérycique des animaux à l'auge. Après avoir vérifié qu'au bout de 2 heures tous les moutons avaient terminé leurs grands repas, nous avons considéré ensuite qu'à ce moment-là la pesée des refus permettait de calculer exactement les quantités ingérées d'ensilage par grands repas.

Pour les fourrages verts, les refus ont été pesés au bout de 2,5 à 3 heures, compte tenu des observations faites au cours de l'étude de Dulphy \& Bechet (1976).

Des échantillons frais d'ensilage ont été prélevés chaque jour de mesure lors des distributions d'ensilage, durant les 6 jours de la période expérimentale. Ils ont été regroupés en un seul échantillon par ensilage étudié. Sur les jus provenant de chacun de ces échantillons, nous avons déterminé le $\mathrm{pH}$ et les teneurs en ammoniac (CONWAY, 1957), en acide lactique (BARKer \& Summerson, 1941) et en acides gras volatils par chromatographie en phase gazeuse (RIGAud \& JourNET, 1970). Ce sont ces critères qui seront utilisés pour caractériser la qualité de conservation des ensilages.

\section{Résultats}

Pour les 30 ensilages suivis selon la méthode de Ruckebusch (1963), les durées des grands repas ont été respectivement de $46 \mathrm{mn}$ pour les 12 graminées, $66 \mathrm{mn}$ pour les 10 trèfles et $47 \mathrm{mn}$ pour 8 luzernes. Ces durées sont égales environ à la moitié des durées correspondantes pour les fourrages verts $(90$ à 100 minutes pour 43 graminées et 75 minutes pour 5 luzernes étudiées par Dulphy \& BECHET, 1976). Les quantités ingérées correspondantes par grands repas ont été respectivement égales à $10,7-18,1$ et $13,1 \mathrm{~g}$ de MS/kg O",75. Dans l'étude de Dulphy \& Bechet (1976), elles étaient de 22,3 g de MS pour les graminées et 29,9 g pour les luzernes. Enfin les durées unitaires d'ingestion ont été respectivement de $4,30-3,65$ et $3,59 \mathrm{mn} / \mathrm{g} / \mathrm{kg} \mathbf{P}^{0,75}$, soient des valeurs très voisines de celles généralement observées avec les fourrages verts.

Les résultats obtenus sur l'ensemble des 109 ensilages étudiés confirment que les quantités de matière sèche ingérées par grands repas sont faibles avec les ensilages : en moyenne $12,6 \mathrm{~g} / \mathrm{kg} \mathrm{P}^{\mathrm{m}, i}$. Pour les 63 ensilages comparés aux fourrages verts correspondants, elles ont été respectivement de 12,7 , contre $26,1 \mathrm{~g} / \mathrm{kg} \mathrm{P}^{0.75}$, c'est-à-dire près de deux fois plus faibles $(\mathrm{P}<0,01)$.

En valeur relative, l'écart entre ensilage et fourrage vert est un peu plus élevé pour les graminées $(11,1$ contre $24,1 \mathrm{~g} ; \mathrm{n}=36)$ que pour les luzernes $(14,8$ contre $28,8 \mathrm{~g} ; \mathrm{n}=27$ ), soit respectivement des quantités d'ensilage ingérées par grands repas égales à 46 et 60 p. 100 des quantités de fourrage vert ingérées. Les quantités ingérées journalières moyennes correspondantes ont été de 45,5 contre $66,3 \mathrm{~g}$, soit 69 p. 100 pour les graminées prairiales et de 58,8 contre $83,6 \mathrm{~g} / \mathrm{kg} \mathbf{P}^{1} . \pi^{7 \pi}$, soit 70 p. 100 pour les luzernes. 


\section{A. Facteurs de variation des quantités ingérées par grands repas}

\section{Finesse de hachage}

Les quantités de matière sèche ingérées par grands repas ont été peu, mais non significativement, augmentées par le hachage fin des ensilages lors de leur récolte $\left(14,3 \mathrm{~g} / \mathrm{kg} P^{0.75}\right.$ pour 10 ensilages à brins moyens ou longs contre 15,5 pour ceux à brins courts correspondants dont la qualité de conservation était comparable ; + 8 p. 100). Nous avons observé l'inverse avec le hachage à la sortie du silo, mais toujours sans écart significatif $\left(12,8 \mathrm{~g} / \mathrm{kg} \mathrm{P}^{0,75}\right.$ pour 13 ensilages non hachés contre 12,0 pour les mêmes après hachage avec le hache-paille : $-6 \mathrm{p} .100$ ).

Pour 10 fourrages présentés de 3 façons :

- hachés avant la mise en silo ;

- ensilés avec des brins moyens ou longs, mais non hachés à la sortie du silo ;

- ensilés avec des brins moyens ou longs et hachés à la sortie du silo,

les durées d'ingestion par grands repas ont été respectivement de 55-62 et $46 \mathrm{mn}$ et les données unitaires d'ingestion de $3,64-4,30$ et $3,48 \mathrm{mn} / \mathrm{g}$ de $\mathrm{MS} / \mathrm{kg} \mathrm{P}^{0,7}$ i (qi de $15,5-14,3$ et $13,2 \mathrm{~g}$ de $\mathrm{MS} / \mathrm{kg} \mathrm{P}^{(0,75}$ ).

\section{Espèce végétale}

Lors de chacun des deux grands repas d'ensilage, les moutons ont consommé respectivement (en $\mathrm{g}$ de $\mathrm{MS} / \mathrm{kg} \mathrm{P}^{0,75}$ ) :

$18,1 \mathrm{~g}$ de trèfle violet, $14,8 \mathrm{~g}$ de luzerne,

$10,8 \mathrm{~g}$ de graminćes prairiales et $11,8 \mathrm{~g}$ de maïs.

Ces quantités ont représenté seulement 25 p. 100 des quantités ingérées par jour, alors que, lors de chacun des grands repas de fourrage vert, les animaux ont ingéré 36 p. 100 des quantités ingérées par jour.

Pour des qualités de conservation très correctes, il y a donc de gros écarts entre espèces, par exemple entre les ensilages de trèfle (toutes longueurs de brins confondus) et ceux de graminées à brins courts.

Toutes les graminées prairiales ont été regroupées car nous n'avons pas pu mettre en évidence de différences systématiques entre elles.

\section{Qualité de conservation}

Compte tenu de ce qui a été dit plus haut, l'influence de la qualité de conservation ne peut s'étudier qu'à l'intérieur d'une espèce végétale donnée, les graminées prairiales étant cependant regroupées. La finesse de hachage a peu d'effet sur les; quantités ingérées par grands repas, mais a un effet important sur les quantités ingérées par jour. Nous avons donc tenu compte de ce fait pour établir deux catégories d'ensilages de graminces et deux de luzerne : brins courts d'une part, brins moyens et longs de l'autre, cette dernière catégorie incluant les ensilages hachés à la sortie du silo. Dans ces conditions nous avons fait 6 groupes d'ensilages dont les caractéristiques moyennes sont données dans les tableaux 2 et 3 . Notons que les ensilages de maïs étaient particuliers, avec une teneur en matière sèche faible. 
㠃

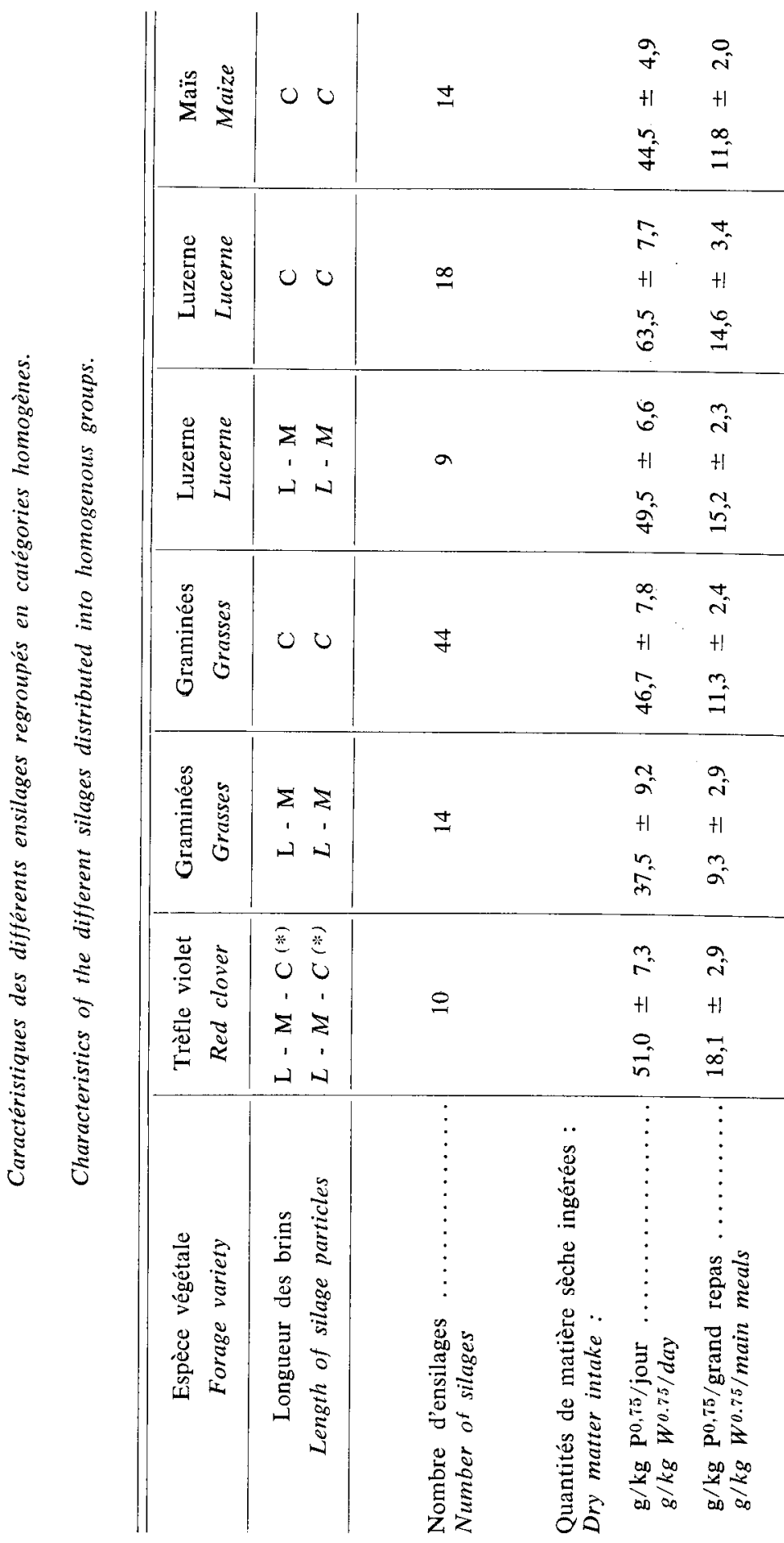




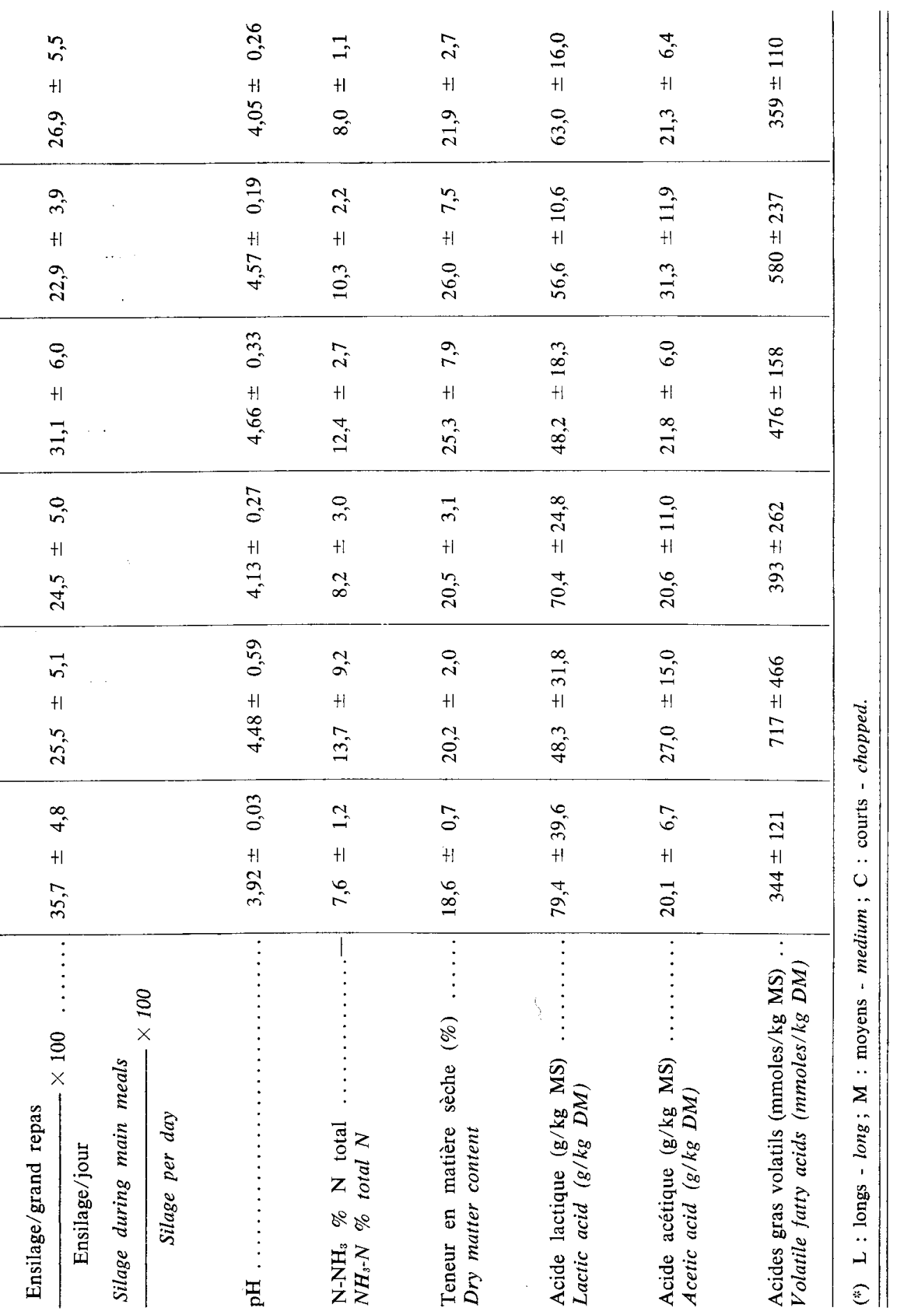




\section{Tableau 3}

Quantités de matière sèche ingérées comparées pour les ensilages et les fourrages verts correspondants.

Comparison between levels of silage and fresh forage DM intake.

\begin{tabular}{|c|c|c|c|c|c|}
\hline & $\begin{array}{l}\text { Trètle violet } \\
\text { Red clover }\end{array}$ & $\begin{array}{c}\text { Graminées } \\
\mathrm{L}+\mathrm{M} \\
\text { Grasses }\end{array}$ & $\begin{array}{c}\text { Graminées } \\
\text { C } \\
\text { Grasses }\end{array}$ & $\begin{array}{l}\text { Luzerne } \\
\mathrm{L}+\mathrm{M} \\
\text { Lucerne }\end{array}$ & $\begin{array}{l}\text { Luzerne } \\
\text { C } \\
\text { Lucerne }\end{array}$ \\
\hline \begin{tabular}{l}
$\qquad \mathrm{n}$ \\
\multicolumn{1}{c}{ Quantités de MS } \\
ingérées/jour: \\
DM intake/day :
\end{tabular} & 10 & 14 & 41 & 9 & 18 \\
\hline $\begin{array}{l}\text { - Fourrage vert } \ldots . . . \\
\text { Fresh forage }\end{array}$ & 64,5 & $61,0 \pm 7,4$ & $63,5 \pm 9,1$ & $84,1 \pm 8,5$ & $83,4 \pm 5,9$ \\
\hline - Ensilage $\ldots \ldots \ldots \ldots$ & $51,0 \pm 7,3$ & $37,5 \pm 9,2$ & $46,4 \pm 8,1$ & $49,5 \pm 6,6$ & $63,5 \pm 7,7$ \\
\hline $\begin{array}{l}\qquad \mathrm{n} \\
\text { Quantités de MS ingérées } \\
\text { par grand repas } \\
\text { DM intake/main meal }\end{array}$ & 0 & 2 & 34 & 9 & 18 \\
\hline $\begin{array}{l}\text { - Fourrage vert } \ldots . . . \\
\quad \text { Fresh forage }\end{array}$ & - & 21,8 & $24,3 \pm 4,2$ & $29,6 \pm 2,4$ & $28,3 \pm 2,1$ \\
\hline - Ensilage $\ldots \ldots \ldots \ldots$ & & 8,5 & $11,3 \pm 2,6$ & $15,2 \pm 2,3$ & $14,6 \pm 3,4$ \\
\hline
\end{tabular}

Pour l'ensemble des 109 ensilages, il existe des liaisons négatives, mais faibles, entre les quantités ingérées par grands repas et les proportions de $\mathrm{N}-\mathrm{NH}_{3}$ dans $\mathrm{N}$ total $(r=-0,224 *)$ ainsi qu'avec les teneurs en acides gras volatils $\left(r=-0,286^{*}\right)$. Par contre, il n'y a pas de liaisons significatives avec le $\mathrm{pH}$, la teneur en matière sèche et en acide lactique. Celle avec l'acide acétique est à la limite de la signification $(r=-0,185)$.

Pour les différentes catégories d'ensilages, les liaisons significatives sont données dans le tableau 4. Les résultats correspondants confirment l'absence d'influence du $\mathrm{pH}$ (sauf pour le trèfle, mais avec une fourchette de $\mathrm{pH}$ très restreinte : 0,1 unité) et de la teneur en acide lactique. La teneur en $\mathrm{N}-\mathrm{NH}_{3}$ dans $\mathrm{N}$ total est le meilleur critère significatif pour les graminées et les luzernes hachées finement. La teneur en matière sèche joue un rôle négatif, de même que les teneurs en acide acétique et en AGV. Les luzernes à brins moyens et longs sont à part (effet positif des AGV) mais elles sont peu nombreuses et leurs teneurs en acide acétique et en AGV sont peu dispersées. 


\section{TableaU 4}

Liaisons entre les quantités ingérées par grands repas et les différents critères caractérisant la qualité de conservation des ensilages.

Relationships between DM intake during the main meals and different silage characteristics.

\begin{tabular}{|c|c|c|c|c|c|c|}
\hline$\therefore$ & $\begin{array}{c}\text { Trèfle } \\
\text { violet } \\
\text { Red clover }\end{array}$ & $\begin{array}{c}\text { Graminées } \\
\mathbf{L}+\mathbf{M} \\
\text { Grasses }\end{array}$ & $\begin{array}{c}\text { Graminées } \\
\mathrm{C} \\
\text { Grasses }\end{array}$ & $\begin{array}{l}\text { Luzerne } \\
\mathbf{L}+\mathbf{M} \\
\text { Lucerne }\end{array}$ & $\begin{array}{l}\text { Luzerne } \\
\text { C } \\
\text { Lucerne }\end{array}$ & $\begin{array}{l}\text { Maïs } \\
\text { Maize }\end{array}$ \\
\hline $\begin{array}{l}\text { Teneur en matière } \\
\text { sèche }(\%) \\
\text { DM content }(\%)\end{array}$ & $\begin{array}{r}-0,644(*) \\
-2,48(* *) \\
64,3(* * *)\end{array}$ & NS & $\begin{array}{c}-0,414 \\
-0,32 \\
17,8\end{array}$ & $\begin{array}{c}-0,739 \\
-0,21 \\
20,6\end{array}$ & NS & NS \\
\hline $\begin{array}{l}\mathrm{pH} \\
p H\end{array}$ & $\begin{array}{l}-0,618 \\
-56,4 \\
239\end{array}$ & NS & NS & NS & NS & NS \\
\hline $\begin{array}{l}\mathrm{N}-\mathrm{NH}_{3} \% \mathrm{~N} \text { total } \\
\mathrm{NH}_{3}-\mathrm{N} \% \text { total } \mathrm{N}\end{array}$ & NS & NS & $\begin{array}{c}-0.534 \\
-0,43 \\
14,8\end{array}$ & NS & $\begin{array}{c}-0,499 \\
-0,75 \\
22,3\end{array}$ & NS \\
\hline $\begin{array}{l}\text { Teneur en acide } \\
\text { lactique } \mathrm{g} / \mathrm{kg} \mathrm{MS} \\
\text { Lactic acid content } \\
\mathrm{g} / \mathrm{kg} \mathrm{DM}\end{array}$ & NS & NS & NS & NS & NS & $\begin{array}{c}-0,413 \\
-0,05 \\
15,1\end{array}$ \\
\hline $\begin{array}{l}\text { Teneur en acide } \\
\text { acétique } \mathrm{g} / \mathrm{kg} \mathrm{MS} \\
\text { Acetic acid content } \\
\text { g/kg DM }\end{array}$ & NS & $\begin{array}{c}-0,657 \\
-0,09 \\
11,8\end{array}$ & $\begin{array}{c}-0,334 \\
-0,07 \\
12,8\end{array}$ & $\begin{aligned}+ & 0,649 \\
+ & 0,25 \\
& 9,8\end{aligned}$ & NS & NS \\
\hline $\begin{array}{l}\text { Teneur en AGV } \\
\text { mmoles } / \mathrm{kg} \text { MS } \\
V F A \text { content } \\
\text { mmoles } / \mathrm{kg} \text { DM }\end{array}$ & $\begin{array}{c}0,606 \\
-\quad 0,014 \\
23,0\end{array}$ & $\begin{array}{c}-0,539 \\
-0,0024 \\
11,0\end{array}$ & $\begin{array}{c}-0,442 \\
-0,004 \\
12,8\end{array}$ & $\begin{array}{l}+0,802 \\
+0,0115 \\
\quad 9,7\end{array}$ & NS & NS \\
\hline $\begin{array}{ll}(*) & \text { Coefficient de } \\
(* *) & \text { Pente de la rég } \\
(* * * *) & \text { Ordonnée à l'o }\end{array}$ & $\begin{array}{l}\text { corrélation - } \mathrm{Co} \\
\text { gression linéaire } \\
\text { origine - Zero-int }\end{array}$ & $\begin{array}{l}\text { orrelation coe } \\
\text { - Linear regi } \\
\text { tercepts. }\end{array}$ & $\begin{array}{l}\text { fficient. } \\
\text { ression slope. }\end{array}$ & & & \\
\hline
\end{tabular}

Pour la catégorie des graminées à brins courts, qui est la plus importante, on améliore peu la relation avec la proportion en $\mathrm{N}-\mathrm{NH}_{3}$ en calculant une équation qui inclue en même temps le $\mathrm{pH}$, les teneurs en $\mathrm{AGV}$, en matière sèche et en acide lactique ( $\mathrm{r}$ passe de 0,534 à 0,575 ). $\mathrm{Si}$ on calcule une relation curvilinéaire en rajoutant $\left(\mathrm{N}-\mathrm{NH}_{3} \text { p. } 100 \mathrm{~N} \text { total }\right)^{2}$, $\mathrm{r}$ passe seulement à 0,604 . Le même type de relation calculée avec les AGV a un coefficient de corrélation de 0,509.

Finalement, même en tenant compte des différentes catégories d'ensilage, la part des variations des quantités ingérées par grands repas, expliquée par les varia- 
tions des paramètres de la qualité de conservation est donc relativement faible : de 15 à 35 p. 100 seulement.

Pour les ensilages de graminées à brins courts (34) et ceux de luzernes (18) pour lesquels nous disposons en plus de la quantité de fourrage vert ingérée par grands repas, il n'y a pas de liaisons significatives entre les quantités d'ensilages ingérées par grands repas et celles ingérées en vert. Par contre, il y en a entre les quantités d'ensilage ingérées par grands repas et la proportion en $\mathrm{N}_{-} \mathrm{NH}_{3}$ p. 100 $\mathrm{N}$ total $(\mathrm{r}=-0,566 *$ et $\mathrm{r}=-0,499 *$ respectivement). Les liaisons avec d'autres critères de qualité de conservation ne sont pas significatives, sauf pour les graminées avec la teneur en $\mathrm{AGV}\left(\mathrm{r}=-0,488^{*}\right)$. Cependant, si on utilise la quantité de fourrage vert ingérée par grand repas comme covariable on obtient les liaisons suivantes :

qi ens $=11,4+0,175$ qif.v. $-0,558 \mathrm{~N}-\mathrm{NH}_{3} \pm 2,1 \quad \mathrm{r}=0,634 * *$ pour les graminées

qi ens $=17,6+0,187$ qif.v. $-0,805 \quad \mathrm{~N}-\mathrm{NH}_{3} \pm 3,1 \quad \mathrm{r}=0,511 *$ pour les luzernes

\section{B. Quantités d'acides organiques ingérées}

Les quantités moyennes et maximales, par espèce végétale, d'acides organiques ingérées par jour sont rapportées dans le tableau 5. Les moutons ingèrent des quantités très variables d'acides organiques selon les ensilages. Dans nos essais, ces quantités ont varié, pour un mouton standard de $60 \mathrm{~kg}$, de 3,7 à $159 \mathrm{~g} /$ jour pour l'acide lactique, de 9,3 à $75 \mathrm{~g} /$ jour pour l'acide acétique, et de 157 à 1539 mmoles/ jour pour les acides gras volatils. Il existe des liaisons linéaires étroites entre ces quantités journalières d'acides organiques ingérées et la teneur de ceux-ci dans les ensilages, ce qui signifie que c'est avec les ensilages les plus riches en acide lactique ou en acides gras volatils que les moutons ingèrent le plus de ces acides.

Les quantités d'acides organiques ingérées par grands repas sont aussi très variables : de 0,4 à $53 \mathrm{~g} /$ repas pour l'acide lactique, de 1,9 à $18 \mathrm{~g} /$ repas pour l'acide acétique, et de 45 à 317 mmoles/repas pour les acides gras volatils. Elles sont également très bien reliées aux teneurs correspondantes dans les ensilages et, là encore, plus les ensilages contiennent de l'acide lactique ou d'acides gras volatils, plus les animaux ingèrent une quantité importante de ces acides.

\section{Discussion}

La principale constatation à laquelle conduisent nos résultats est bien le rassasiement rapide des moutons ingérant des ensilages, et surtout le fait que ce rassasiement rapide existe même pour les ensilages très bien conservés. Ces résultats confirment ceux de Dulphy, Bechet \& Thomson (1975) puis de Dulphy, Michalet-Doreau \& Demarquilly (1984) et montrent qu'on observe ce phénomène non seulement pour les graminées fourragères, mais encore pour des ensilages de maïs relativement humides, mais bien conservés, ainsi que pour les légumineuses, ces dernières s'ingérant cependant nettement plus que les graminées lors des grands repas. 


\section{TABLead 5}

Quantités d'acides organiques ingérées par jour et par grands repas (pour un mouton standard de $60 \mathrm{~kg}$ ).

Organic acid intake per day and during each main meal (for a $60 \mathrm{~kg}$ standard sheep).

\begin{tabular}{|c|c|c|c|c|c|c|c|}
\hline & \multirow{2}{*}{$\begin{array}{l}\text { Espèce végétale } \\
\text { Forage species }\end{array}$} & \multicolumn{2}{|c|}{$\begin{array}{l}\text { Acide lactique } \\
\mathrm{g} / \text { mouton } \\
\text { Lactic acid } \\
\text { g/sheep }\end{array}$} & \multicolumn{2}{|c|}{$\begin{array}{l}\text { Acide acétique } \\
\mathrm{g} / \text { mouton } \\
\text { Acetic acid } \\
\text { g/sheep }\end{array}$} & \multicolumn{2}{|c|}{$\begin{array}{l}\text { Acides gras volatils } \\
\text { mmoles/mouton } \\
\text { Volatile fatty acid } \\
\text { mmoles/sheep }\end{array}$} \\
\hline & & $\begin{array}{c}\text { Moyenne } \\
\text { Mean }\end{array}$ & $\begin{array}{l}\text { Maxi- } \\
\text { mum } \\
\text { Maxi- } \\
\text { mum }\end{array}$ & $\begin{array}{c}\text { Moyenne } \\
\text { Mean }\end{array}$ & $\begin{array}{l}\text { Maxi- } \\
\text { mum } \\
\text { Maxi- } \\
\text { mum }\end{array}$ & $\begin{array}{c}\text { Moyenne } \\
\text { Mean }\end{array}$ & $\begin{array}{l}\text { Maxi- } \\
\text { mum } \\
\text { Maxi- } \\
\text { mum }\end{array}$ \\
\hline \multirow{4}{*}{$\begin{array}{c}\text { Quantités } \\
\text { ingérées } \\
\text { par jour } \\
\text { Daily intake }\end{array}$} & $\begin{array}{l}\text { Graminées ... } \\
\text { Grasses }\end{array}$ & 63,8 & 159 & 20,5 & 72 & 414 & 1356 \\
\hline & $\begin{array}{l}\text { Trèfle violet .. } \\
\text { Red clover }\end{array}$ & 86,5 & 150 & 21,8 & 30 & 371 & 515 \\
\hline & $\begin{array}{l}\text { Luzerne } . . . \\
\text { Lucerne }\end{array}$ & 68,6 & 106 & 36,9 & 75 & 709 & 1539 \\
\hline & $\begin{array}{l}\text { Maïs } \\
\text { Maize }\end{array}$ & 61,2 & 91 & 20,7 & 26 & 349 & 668 \\
\hline \multirow{4}{*}{$\begin{array}{c}\text { Quantités } \\
\text { ingérées } \\
\text { par grands repas } \\
\text { Intake during } \\
\text { a main meal }\end{array}$} & $\begin{array}{l}\text { Graminées } \ldots \\
\text { Grasses }\end{array}$ & 15,3 & 39 & 4,7 & 12 & 99 & 317 \\
\hline & $\begin{array}{l}\text { Trèfle violet .. } \\
\text { Red clover }\end{array}$ & 30,0 & 53 & 7,8 & 12 & 129 & 196 \\
\hline & $\begin{array}{l}\text { Luzerne ...... } \\
\text { Lucerne }\end{array}$ & 17,0 & 31 & 8,8 & 18 & 172 & 302 \\
\hline & $\begin{array}{l}\text { Maïs ... } \\
\text { Maize }\end{array}$ & 15,7 & 25 & 5,4 & 9 & 91 & 144 \\
\hline
\end{tabular}

Par rapport aux conclusions de Forbes (1979), l'apport d'acide propionique ne peut être effectivement mis en cause car les ensilages d'herbe en contiennent très peu. Par contre, l'intervention d'autres signaux est à envisager. Nous la discuterons d'abord pour les ensilages bien conservés, puis pour les autres.

Les ensilages bien conservés contiennent peu d'acide acétique et pratiquement pas d'autres acides gras volatils. Par contre, ils contiennent des quantités élevées d'acide lactique et leur $\mathrm{pH}$ est faible. Or on sait que l'acide lactique, apporté sur des fourrages secs fait baisser les quantités journalières ingérées : - 13 p. 100 pour un apport de $75 \mathrm{~g} / \mathrm{j}$ environ chez des moutons (Morgan \& L'Estrange, 1976). Un effet négatif sur les quantités ingérées a été observé aussi par McLEOD, WILKINs \& RAYMond (1970). Par contre il semble que l'apport de lactate ait peu d'effet 
(Thomas et al., 1961 ; Senel \& OWen, 1966). La neutralisation de l'acide lactique peut même augmenter l'ingestion (MCLEOD, WILKINS \& RAYMOND, 1970). Dans ces essais, l'effet de l'acide lactique sur les quantités ingérées par grands repas n'a pas été noté.

Pour les ensilages bien conservés, qui contiennent une quantité notable d'acide lactique, on pourrait donc faire l'hypothèse que l'apport élevé d'acide lactique explique le faible niveau d'ingestion lors des grands repas. L'acide lactique est assez vite métabolisé (Morgan \& L'Estrange, 1976) ce qui permettrait ensuite aux animaux d'ingérer plus d'ensilage que de fourrage vert lors des petits repas et de rattraper partiellement leur «handicap». En ce qui concerne le mécanisme d'action on ne peut évidemment rien conclure de nos observations, mais Bueno (1975) a montré que l'acide lactique arrivant au niveau du duodénum est un facteur de rassasiement important.

On sait par ailleurs que les bovins sont à la fois moins sensibles à l'acide lactique que les ovins (MORGAN \& L'Estrange, 1970) et qu'ils ne présentent pas, avec les bons ensilages, le phénomène de rassasiement rapide (Dulphy, Michalet-Doreau \& Demarquilly, 1984). Cela pourrait conforter l'hypothèse avancée plus haut pour les moutons.

Pour ces ensilages bien conservés, l'incidence de la distension du rumen n'est cependant pas à exclure. En effet, le remplissage du rumen est la résultante entre l'arrivée de fourrage et le départ d'une partie du contenu vers l'intestin grêle. L'ingestion d'ensilage finement haché est rapide, plus rapide que celle du fourrage vert de départ (Dulphy, Michalet-Doreau \& Demarquilly, 1984) et il peut y avoir un ralentissement du transit par l'apport important d'acides dans le rumen (MrchaletDoreau, 1975). Cela pourrait expliquer les niveaux élevés de contenu de rumen en fin de grands repas pour de très bons ensilages (Dulphy, Bechet \& Thomson, 1975), mais, n'ayant pas étudié ici ce qui se passait dans le rumen, nous ne discuterons pas plus cette éventualité.

Pour les autres ensilages, au fur et à mesure que leur qualité de conservation se dégrade, l'effet de la teneur en acide lactique doit être de plus en plus faible. II n'est d'ailleurs statistiquement pas significatif. Par contre, on note un effet négatif sur les quantités ingérées par grands repas des teneurs en ammoniac et en acides gras volatils, en particulier de l'acide acétique qui est le plus abondant. Pour des ensilages mal conservés, les quantités ingérées par grands repas sont alors en général diminuées, bien que les résultats soient très dispersés, et passent, en moyenne, pour les graminées fourragères, au-dessous de $10 \mathrm{~g}$ de $\mathrm{MS} / \mathrm{kg} \mathbf{P}^{0,75}$ contre 12-15 avec les ensilages bien conservés. Cet effet n'est pas aussi évident pour les ensilages de luzerne dont les ensilages à brins longs mal conservés ont des quantités ingérées par grands repas élevés, quoique très inférieures à celles ingérées en vert. Quels sont alors les mécanismes mis en place? Plusieurs possiblités existent :

- au niveau sensitif, la possiblité d'une répulsion olfactive ou gustative pour les ensilages mal conservés (Michaler-Doreau, 1975). D'ailleurs, Gatel (1984) a bien montré qu'avec des fourrages secs l'appétence était un facteur très important pour expliquer la rapidité ou non de l'établissement de la satiété à court terme ;

- au niveau ruminal, l'intervention de composés organiques tels que l'acide acétique sur des récepteurs chimio-sensibles (Baile \& Pfander, 1966; Baile \& Mclaughlin, 1970). Cette intervention est très probable car les quantités d'acide 
acétique ingérées par repas sont tout à fait comparables à celles utilisées par ces auteurs ;

- au niveau de la veine porte, l'intervention de composés tel que l'acide propionique (BAILE, 1971), mais les ensilages, même mal conservés contiennent peu de cet acide ;

- au niveau duodénal, l'intervention de certains composés tel que l'acide isobutyrique (Bueno \& RUCKebusch, 1974) et aussi l'acide lactique (BuENo, 1975) encore présent dans beaucoup d'ensilages.

Il est tout à fait probable que, selon les ensilages, plusieurs mécanismes puissent intervenir ensemble, les uns renforçant les autres. Mais le plus frappant c'est l'ampleur du phénomène de rassasiement (en quantité ingérée), en particulier pour les bons ensilages et le temps court au bout duquel il intervient. Il y a donc bien un effet global des acides organiques et les quantités ingérées de ces derniers dépendent avant tout de leur teneur, les grands repas étant arrêtés au bout d'un temps peu variable. Dans un article récent Shaver et al. (1985) parlent d'un effet global du pH sur l'ingestion des ensilages et pensent que tous les ions $\mathrm{H}^{+}$jouent le même rôle quelle que soit leur origine.

On pourrait donc admettre pour l'instant que l'animal réagit avant tout à l'acidité des ensilages, des actions spécifiques de tel ou tel acide venant ensuite amplifier le phénomène de rassasiement observé. Il sera alors nécessaire de compléter la présente étude par l'observation de moutons ingérant des ensilages normalement fermentés, mais additionnés d'un tampon pour ramener leur pH entre 6 et 7 , et des ensilages peu fermentés, c'est-à-dire préfanés.

Le problème qui se pose enfin est de savoir dans quelle mesure le rassasiement rapide des moutons ingérant des ensilages d'herbe limite les quantités ingérées journalières. Ce travail n'était pas destiné à répondre à cette question que nous avons déjà discutée par ailleurs (Dulphy, Michalet-Doreau \& Demarquilly, 1984). On notera cependant que les quantités d'acides organiques ingérées par jour sont très variables, comme nous l'avions déjà constaté (Dulphy \& Demarquilly, 1972). Pour l'acide acétique, les maxima correspondent à ceux cités par Hutchinson \& WiLKins (1971), soit $73 \mathrm{~g} /$ jour pour un mouton de $60 \mathrm{~kg}$. Nous confirmons donc la conclusion de ces auteurs, à savoir que la présence d'acide acétique en elle-même ne semble pas limiter les quantités journalières ingérées. Nos mesures montrent qu'il en est de même pour l'acide lactique, ce qui rejoint les observations de Deswysen (1980). Les moutons, lorsqu'ils sont habitués, peuvent donc ingérer des quantités d'acides organiques considérables, les quantités les plus élevées étant d'ailleurs absorbées avec des ensilages de qualité moyenne à excellente.

Il n'y a donc pas de liens systématiques entre ce qui se passe au cours des grands repas et au cours de la journée. Ceci démontre que l'action de certains facteurs peut être importante, mais fugace, ce qui permet aux animaux de compenser leur rassasiement rapide lors des grands repas par une augmentation des quantités ingérées lors des petits repas en cours de journée. Reste à savoir pourquoi cette compensation n'est pas complète chez les moutons, alors qu'elle peut l'être chez les bovins (DuLPHY, Michalet-Doreau \& Demarquilly, 1984), la constatation d'une moindre sensibilité à l'acide acétique de ces derniers (DESWYSEN, 1980) n'apportant pas une explication suffisante.

Reçu en février 1982.

Accepté en aô̂t 1985. 


\section{Summary}

Level of dry matter intake per main meal in sheep fed forage silages

Using 109 silages (grasses, red clover, lucerne and maize - table 1) we measured the level of dry matter intake in sheep during the two main meals. During these meals the level of intake was low : $10.8 \mathrm{~g} / \mathrm{kg} \mathrm{W} \mathbf{~ W}^{0.75}$ for grasses, 18.1 for red clover, 14.8 for lucerne and 11.8 for maize (table 2). These values represented 40 to $60 \mathrm{p}$. 100 of those of the corresponding fresh forages (table 3), while the rate of intake (especially that of precision chopped silage) did not seem to be changed relative to fresh forages.

For similar conservation qualities, chopping fineness only slightly affected the level of intake during these meals. When ammonia or acetic acid levels increased the levels of intake slowly decreased (table 4). Nevertheless, sheep were able to eat high levels of acids (table 5).

The results were discussed, first considering those of well preserved silages in which lactic acid might account for the rapid satiety observed in the animals. When the conservation quality decreased, the increase in volatile fatty acids, particularly that of acetic acid, might explain the enhanced satiety phenomenon with a possible action of fermentation products on taste and flavour and/or of ruminal and duodenal chemical receptors.

The rapid satiety observed in sheep may partly explain the lower level of silage intake as compared to fresh forage intake. The animals were however able to compensate for this by eating more silage than fresh forage during the small meals. This indicates that some factors acting during the main meals have a smaller effect when considering the whole day.

\section{Références bibliographiques}

BAILE C.A., 1971. Metabolites as feedbacks for control of feed intake and receptor sites in goats and sheep. Physiology and behaviour, 7, 819-826.

Baile C.A., Della-Ferra M.A., 1981. Nature of hunger and satiety control systems in ruminants. J. Dairy Sci., 64, 1140-1152.

BAile C.A., Forbes M.J., 1974. Control of feed intake and regulation of energy balance in ruminants. Physiol. Rev., 54, 160-214.

BaIle C.A., MaYer J., 1969. Depression of feed intake of goats by metabolites injected during meals. Am. J. Physiol., 217, 1830-1836.

Baile C.A., Mc Laughlin C.L., 1970. Feed intake of goats during volatile fatty acid injections into four gastric areas. J. Dairy Sci., 53, 1058-1063.

Baile C.A., Pfander W.H., 1966. A possible chemosensitive regulator mechanism of ovine feed intake. Am. J. Physiol., 210, 1243-1248.

Balch C.C., Campling R.C., 1962. Regulation of voluntary food intake in ruminants. Nutr. Abstr. Rev., 32, 669-686.

BARKER J.B., SUMmerson W.H., 1941. The colorimetric determination of lactic acid in biological material. J. Biol. Chem., 137, 535.

Bueno L., 1975. Rôle de l'acide DL-lactique dans le contrôle de l'ingestion alimentaire chez le mouton. Ann. Rech. Vet., 6, 325-336.

Bueno L., Ruckebusch Y., 1974. Contrôle de l'ingestion alimentaire par des chemorécepteurs duodénaux chez les ruminants. C.R. Acad. Sci., Paris, 279, 409-412 D.

Conway E.J., 1957. In : Microdiffusion analysis and volumetric error. Crosby, Lockwood, London, VIII, $468 \mathrm{pp}$.

Demarquilly C., 1973. Composition chimique, caractéristiques fermentaires, digestibilité et quantités ingérées des ensilages : modification par rapport au fourrage vert initial. Ann. Zootech., 22, 1-35. 
DESWYSEN A., 1980. Influence de la longueur des brins et de la concentration en acides organiques des ensilages sur l'ingestion volontaire chez les ovins et les bovins. Thèse doctorat. Université Catholique de Louvain, mai, 254 p.

Dulphy J.P., 1979. The intake of conserved forages. In : Forage conservation in the 80's. Proc meeting of the European Grassland Federation, Brighton, 27-29 november. C. Thomas Ed., 107-121.

Dulphy J.P., Bechet G., 1976. Influence du stade de végétation et de l'espèce végétale sur le comportement alimentaire et mérycique de moutons recevant des fourrages verts hachés. Ann. Zootech., 25, 505-519.

Dulphy J.P., Bechet G., Thomson E., 1975. Influence de la structure physique et de la qualité de conservation des ensilages de graminées sur leur ingestibilité. Ann. Zootech., 24, $81-94$.

Dulphy J.P., Demarquilly C., 1972. Influence de la finesse de hachage des ensilages de graminées sur le comportement alimentaire des moutons. Ann. Zootech., 21, 443-449.

Dulphy J.P., Demarquilly C., 1973. Influence de la machine de récolte et de la finesse de hachage sur la valeur alimentaire des ensilages. Ann. Zootech., 22, 199-217.

Dulphy J.P., Michalet-Doreau B., Demarquilly C., 1984. Etude comparée des quantités ingérées et du comportement alimentaire et mérycique d'ovins et de bovins recevant des ensilages d'herbe réalisés selon différentes techniques. Ann. Zootech., 33, 291-320.

Dulphy J.P., Remond B., Theriez M., 1979. Ingestive behaviour and related activities in ruminants. In : Digestive Physiology and Metabolism in Ruminonts. Ruckebusch Y. and Thivend P., Ed. M.T.P. Press, p. 103-122.

Forbes J.M., 1979. Hormones and metabolites in the control of feed intake. In : Digestive physiology and Metabolism in Ruminants. RuCKebusch Y. and THIVEND P. Ed. M.T.P. Press, p. 145-160.

Gatel F., 1984. Signification de la satiété à court terme chez le mouton : influence de la qualité du fourrage et des stimuli associés à la prise de nourriture. Ann. Zootech., 33, 111-118.

Hutchinson K.J., Wilkins R.J., 1971. The voluntary intake of forage by sheep. II. The effects of acetate on silage intake. J. Agric. Sci. Camb., 77, 539-544.

MCLeOd D.S., WiLkins R.J., RAYMond W.F., 1970. The voluntary intake by sheep and cattle of silages differing in free-acid content. J. Agric. Sci. Camb., 75, 311-319.

Michalet-Doreau B., 1975. Recherches sur les causes de variations des quantités d'ensilage d'herbe ingérées par les ruminants. Thèse de docteur-ingénieur. Université de Nancy, $99 \mathrm{pp}$.

Morgan D.J., L'Estrange J.L., 1976. Effect of dietary additions of hydrochloric and lactic acid on feed intake and metabolism of sheep and cattle. Ir. J. Agric. Res., 15, 55-63.

Rigaud J., Journet M., 1970. Méthode de dosage des acides gras volatils dans le liquide du rumen. Ann. Biol. anim. Biochim. Biophys., 10, 151-157.

Ruckebusch Y., 1963. Recherches sur la régulation centrale du comportement alimentaire chez les ruminants. Thèse de Doc. Sci. Université de Lyon, 213 p.

SENEL S.H., OWEn F.G., 1966. Relation of dietary acetate and lactate to dry matter intake and volatile fatty acid metabolism. $J$. Dairy Sci., 49, 1075-1079.

Shaver R.D., ERdman R.A., O'Connor A.M., Vandersall J.H., 1985. Effects of silage pH on voluntary intake of corn silage and alfalfa haylage. J. Dairy Sci., 68, 338-346.

Thomas J.W., Moore L.A., Окамотo M., Srkes J.F., 1961. A study of factors affecting rate of intake of heifers fed silage. J. Dairy Sci., 44, 1471-1483.

Wilkins R.J., Hutchinson K.J., Wilson R.F., Harris C.E., 1971. The voluntary intake of silage by sheep. I. Interrelationships between silage composition and intake. J. Agric. Sci., Camb., 77, 531-537. 